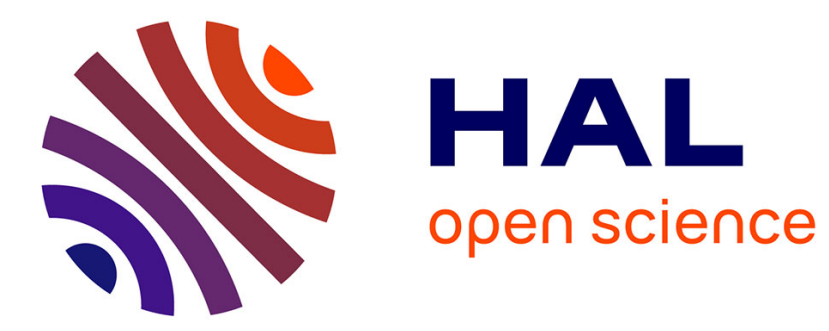

\title{
QUELQUES REMARQUES SUR LE STÉRILISATEUR NIELSEN
}

\author{
Otakar Laxa
}

\section{To cite this version:}

Otakar Laxa. QUELQUES REMARQUES SUR LE STÉRILISATEUR NIELSEN. Le Lait, 1930, 10 (92), pp.122-128. hal-00894983

\section{HAL Id: hal-00894983 \\ https://hal.science/hal-00894983}

Submitted on 1 Jan 1930

HAL is a multi-disciplinary open access archive for the deposit and dissemination of scientific research documents, whether they are published or not. The documents may come from teaching and research institutions in France or abroad, or from public or private research centers.
L'archive ouverte pluridisciplinaire HAL, est destinée au dépôt et à la diffusion de documents scientifiques de niveau recherche, publiés ou non, émanant des établissements d'enseignement et de recherche français ou étrangers, des laboratoires publics ou privés. 


\title{
QUELQUES REMARQUES SUR LE STÉRILISATEUR NIELSEN
}

\author{
par M. le Professeur O. LAXA.
}

Ce fut un grand évènement dans la pratique de la stérilisation du lait quand on a commencé à effectuer celle-ci simultanément en l'a bsence de l'air et à la température élevée de 130-140 C. Ce sont Tödt en Allemagne et Jonas Nielsen au Danemark qui ont appliqué pour la première fois ces prinoipes avec succès dans la laiterie.

La laiterie de Radlice à Smichov à Prague possède un stérilisateur de Nielsen depuis 1926. En 1928, on y a installé une nouvelle machine perfectionnée par le principe régénératif. Le débit de chaque stérilisateur est environ de 6.000 à 9.000 litres par heure. Grâce à l'amabilité du Directeur général Fr. Willinger, j'ai eu la possibilité d'étudier le fonctionnement de la machine au point de vue microbiologique.

Si l'on observe la machine de Nielsen, modèle 1926, on voit que sur le bâti solide reposent six cylindres (fig. 1 et 2 ). Dans chaque cylindre se trouvent 30 tuyaux de nickel qu'on peut enlever et qui communiquent entre eux aux extrémités des cylindres de façon à former un serpentin, le lait pouvant ainsi circuler dans les deux sens. L'espace compris entre les tuyaux dans les cylindres est rempli, suivant le cas, d'eau chaude, de vapeur ou d'eau froide. Le lait coule du bassin dans le récipient $R$ par un tuyau muni d'un robinet avec flotteur. Une pompe centrifuge $\mathrm{L}$ oblige le lait à pénétrer dans le cylindre I où il est chauffé à $60-70^{\circ} \mathrm{C}$. par l'eau condensée; puis par le tuyau $a_{1}$ il passe dans le cylindre II. Dans celui-ci arrive la vapeur directe $(V)$ dont la pression est réglée par une soupape de façon à avoir une température de $108-110^{\circ} \mathrm{C}$. ou $128-130^{\circ} \mathrm{C}$. Ici, le lait atteint la plus haute température en l'absence de l'air. La température est réglée grâce à un petit vase de régulation automatique à l'alcool. La vapeur se condense dans le cylindre et l'eau condensée qui s'écoule dans un pot de fer (P) est reprise par une pompe centrifuge (E) qui l'envoie dans les cylindres I et III. Le passage du lait dans le cylindre II ne dure que 50 secondes et le lait coule par le tuyau $a_{2}$ dans le cylindre III où il est refroidi par l'eau condensée jusqu'à la température de $90^{\circ} \mathrm{C}$. L'eau du eylindre chauffée par le lait peut être utilisée pour le chauffage du lait frais ; à cet effet, la pompe centrifuge l'envoie dans le eylindre I. Par le tuyau ascendant $a_{3}$ le lait entre dans le cylindre IV qui est refroidi par l'eau froide, et sa température s'abaisse à $35^{\circ} \mathrm{C}$. On peut admettre que le lait a été maintenu à la température de $128-130^{\circ}$ pendant une minute, et à 90-108 pendant plus longtemps encore. Le cylindre suivant $V$ dans lequel le lait est amené par le tuyau ascendant $a_{4}$ sert aussi $\mathrm{au}$ refroidissement du lait par l'eau froide, ce qui l'amène à $20^{\circ} \mathrm{C}$. Enfin, le lait, par le tuyau $a_{5}$ passe dans le dernier cylindre VI dans 
lequel cirsule une solution saline refroidie, ce qui abaisse la température à $9^{\circ} \mathrm{C}$., et par le tuyau $m$ le lai $\ell$ sort de la machine.

Tous les tuyaux ascendants sont munis de thermomètres et le oylin. dre II d'un manomètre.

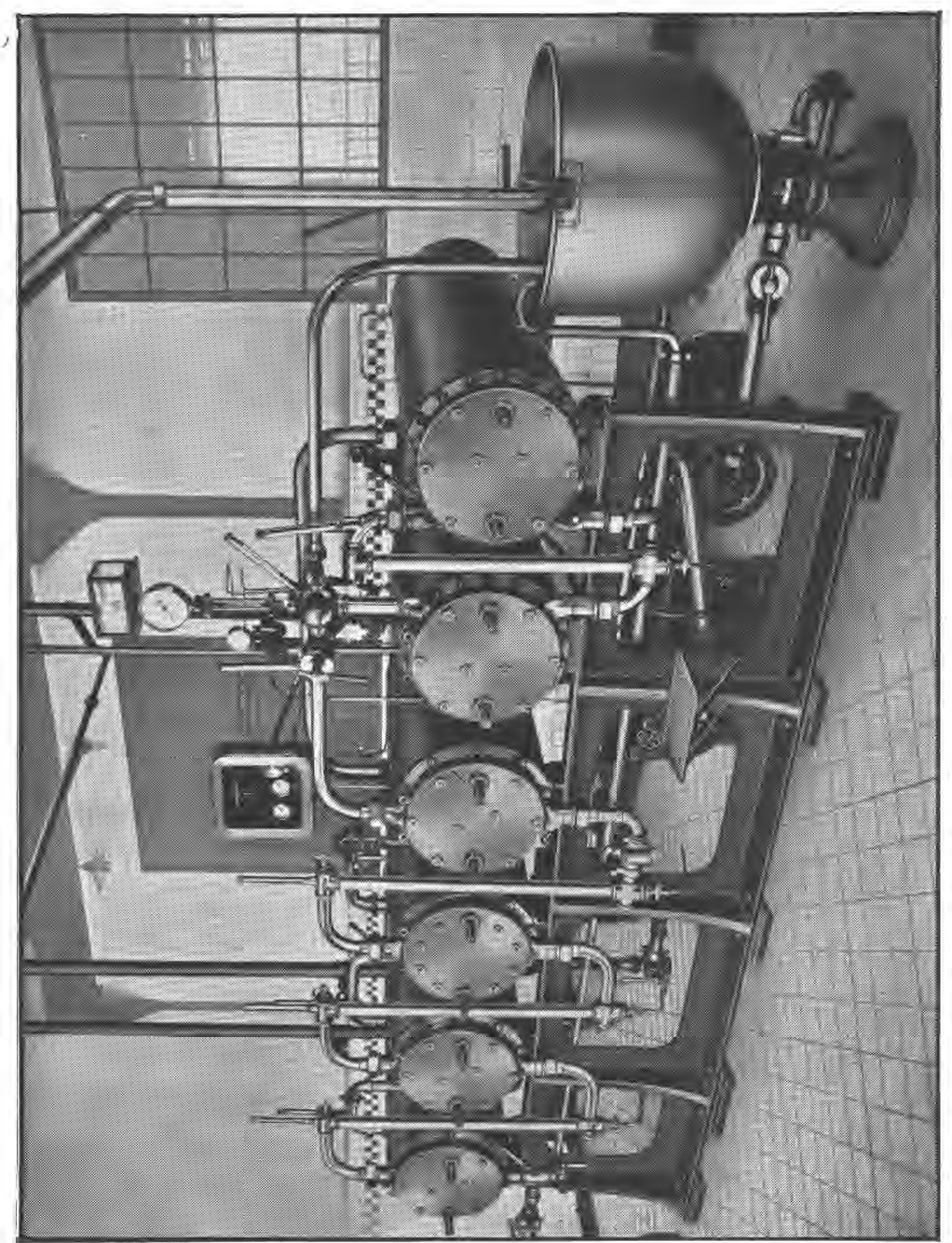

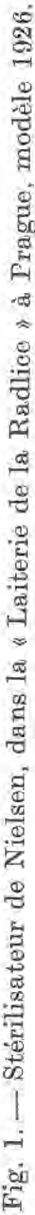

La machine est construite également pour une circulation fermée du lait, et aussi pour le nettoyage des tuyaux.

Au début du travail, alors que les eylindres ne sont pas encore chauffés, on emploie la circulation $n^{a} 1$ : le lait aspiré par la pompe $\mathrm{L}$ passe dans les cylindres $I$ et $I I$ et par le dispositif $c_{1}$ retourne dans le récipient. Dans ce cas, l'eau de condensation ne chauffe que le cylindre I, 


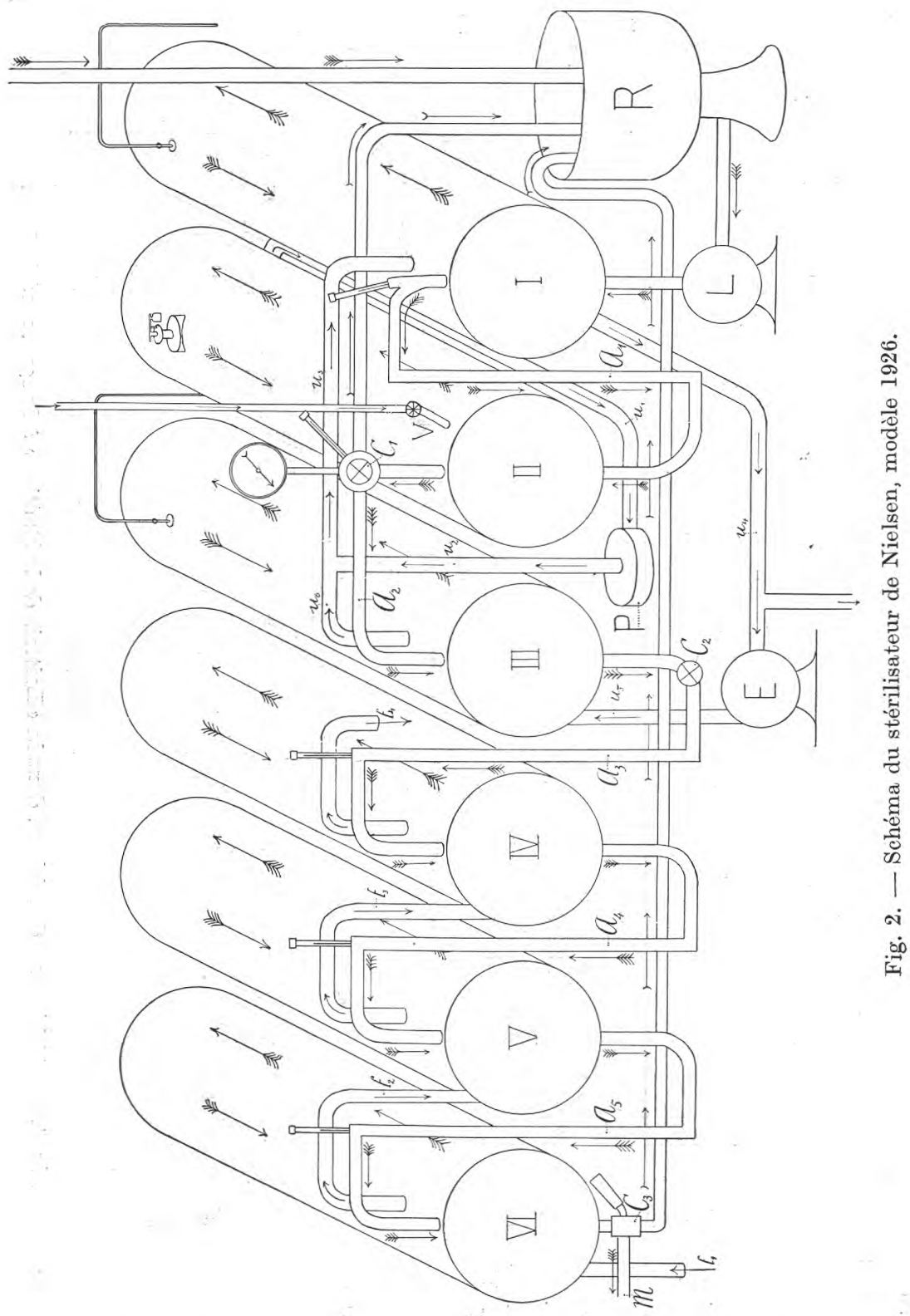

et le cylindre III est plus chaud qu'il n'est désirable. Pour éviter cet inconvénient, on a, à la laiterie de Radlice, utilisé la circulation $\mathrm{n}^{0} 2$ : le lait traverse la pompe et les cylindres I, II et III, et par le dispositif $c_{2}$ 
retourne au récipient. On peut aussi utiliser la circulation $n^{\circ} 3$ par la pompe et tous les cylindres; par le dispositif $c_{3}$, on obtient le retour au récipient. Le nettoyage de la machine se fait aussi par une circulation. On adopte la manière $n^{0} 1$ pour le lavage préalable et la manière $n^{0} 3$ pour le lavage à fond.

Plus tard, Nielsen a perfectionné la machine par le principe régénératif. Je nombre des cylindres est augmenté (fig. 3). Le lait entre dans le cylindre $I$ où on le chauffe au moyen du lait chaud, ce qu'on répète dans les cylindres II, III et IV. Le lait ainsi chauffé à $70^{\circ} \mathrm{C}$., passe dans le cylindre $V$ où la vapeur directe élève sa température à $108-110^{\circ} \mathrm{C}$. ou 128-130 C., et rentre dans les cylindres IV, III, II et I en apportant sa chaleur au lait frais. Ainsi refroidi à $70^{\circ} \mathrm{C}$, , le lait traverse les cylindres VI et VII refroidis à l'eau froide jusqu'à $20^{\circ} \mathrm{C}$. et entre enfin dans le cylindre VIII refroidi par une solution saline; il en sort à la température de $8 \circ \mathrm{C}$.

On a contrôlé l'effet de la stérilisation par l'observation bactériologique en utilisant la numération du nombre de germes cultivés sur plaque de gélatine du petit-lait. On a recherché aussi les germes de Bacterium coli commune au moyen de gelose de Drigalski. Le lait chauffé à 108 $110^{\circ} \mathrm{C}$. dans le cylindre $\mathrm{V}$, modèle 1928, possédait de 12 à 207 germes par $\mathrm{em}^{3}$ qui appartenaient généralement aux espèces de Bacillus mesentericus, mais le lait du cylindre III où régnait une température de $90^{\circ} \mathrm{C}$. n'accusait que 2 à 4 germes dans $1 \mathrm{~cm}^{3}$, et même dans un cas, 1 germe. Vu que ce germe était Sarcina lutea qui ne forme pas de spores et qui est très délicat à l'influence de la chaleur, on peut soupçonner que ce germe était tombé dans le lait pendant le prélévement de l'échantillon et que le lait était stérile. Dans le cas où le lait a été chauffé à $128^{\circ} \mathrm{C}$., on ne pouvait trouver aucun microorganisme ; il était stérile.

Il résulte de ces observations que si l'on chauffe le lait à $108-110^{\circ} \mathrm{C}$., on n'atteint pas l'anéantissement de tous les microbes dans le cylindre $V$, mais dans le cylindre III. Il faut donc un chauffage prolongé pour pouvoir atteindre le même effet que par le chauffage à 128-130 C. L'étude du nombre des germes dans le lait des cylindres réfrigérants où il est à des températures de $40,14,11$ et $8^{\circ} \mathrm{C}$. a confirmé le fait que pour maintenir la stérilisation, il faut nettoyer les cylindres d'une manière efficace, et tenir la température à au moins $90^{\circ} \mathrm{C}$. pendant le nettoyage final. Après chaque stérilisation, on lave les tuyaux des cylindres à l'cau chaude additionnée de soude et de silicate de soude; cette dernière solution dépose de la silice sur les parois des tuyaux. C'est pourquoi cn cuvre les fonds des cylindres et on enlève le dépôt avec un pincєau métalliçve. On ferme les cylindres et on met la machine en foncticn $\in \mathbf{n}$ craufant l'€eu à $90^{\circ} \mathrm{C}$. au lieu du lait. Si l'on néglige cette précauticn, le lait circula nt dans les cylindres réfrigérants contient jusqu'à 1.000 germ€s par $\mathrm{cm}^{3}$. Si, au contraire, les cylindres réfrigérants ont été bien stérilisés pendant le 
nettoyage, on ne trouve dans le lait chauffé à $109^{\circ} \mathrm{C}$. que 10 germes, et dans le lait chauffé à $128^{\circ} \mathrm{C}$. aucune germe par $\mathrm{cm}^{3}$.

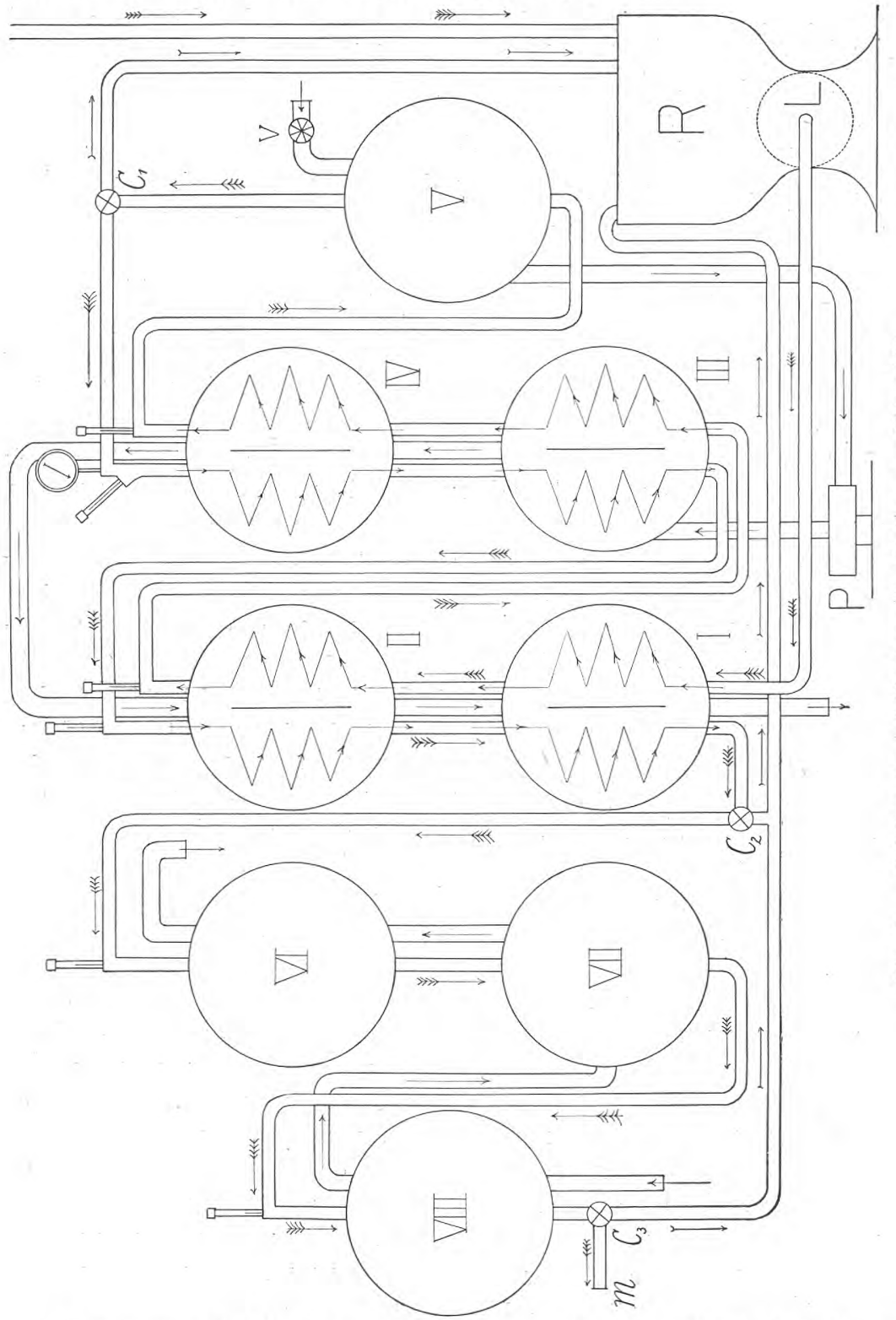

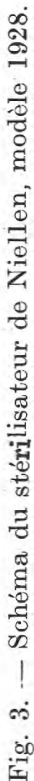

La présence de Bacterium coli commune dans le lait stérilisé dépend aussi du degré de chauffage des cylindres réfrigérants pendant le nettoyage. Tous les germes de cette bactérie périssent dans les cylindres chauffés à 
$90^{\circ} \mathrm{C}$., mais si les cylindres ne sont pas bien stérilisés pendant le nettoyage, les germes de $B$. coli commune entrent par les robinets; le lait peu refroidi est favorable à leur végétation, et le lait déjà stérilisé s'infecte.

Il est évident que non seulement le nombre des germes, mais aussi la conservation du lait, dépend de la température employée pour le chauffage et du nettoyage plus ou moins parfait de la machine. Le lait stérilisé à $108^{\circ} \mathrm{C}$, a vec un chauffage insuffisant des cylindres réfrigérants lors du nettoyage, ne se conservait dans l'étuve à $37^{\circ} \mathrm{C}$. que 2 à 4 jours; au bout de ce temps, il tournait et changeait par la peptonisation. Le lait stérilisé à $128^{\circ} \mathrm{C}$. a près chauffage efficace des cylindres réfrigérants ne changeait pas dans l'étuve à $37^{\circ} \mathrm{C}$. pendant 15 jours, et l'on n'a pu reconnaître aucun changement d'aspect, même par le goût et l'odeur. Il faut remarquer que les observations sur la conservation du lait n'ont pas été faites avec une petite quantité de lait, mais on a pris dans chaque cas 10 , 30,100 et $300 \mathrm{~cm}^{3}$, et on a trouvé que les modifications se présentaient de la même manière dans tous les échantillons, ce qui prouve que la machine chauffe le lait d'une manière parfaite.

Il faut tenir grand compte du fait que le lait à stériliser ne doit pas contenir d'ésume. L'ésoulement violent du lait dans le récipient peut avoir comme conséquence que ce lait contient beaucoup d'air, lequel porté à haute température produit une modification désagréable du goût. Si l'on néglige cette précaution, on trouve au bout de temps variables, un lait ayant le goût du saindoux, ou même de l'huile ; on peut expliquer ceci par l'influence de l'oxygène de l'air sur l'oléine de la graisse du lait, La présaution d'enlever l'écume du lait avant la stérilisation est exigée aussi par le fait que le chauffage doit être effectué en l'absence de l'air.

La question est de savoir si le lait stérilisé est de meilleure qualité que le lait pasteurisé à la température de $85^{\circ} \mathrm{C}$. Les eauses suivantes portent à préférer la stérilisation à la pasteurisation à haute température :

10 Par la pasteurisation à $85^{\circ} \mathrm{C}$., les germes sont détruits dans la proportion de $99,5 \%$, mais il reste les spores des bactéries nuisibles, et même celles de la fermentation butyrique, des bactéries de la putréfaction (Bacillus putrificus) et des bactéries d'espèce B. Subtilis et B. mesentericus. La stérilisation à $128-130^{\circ} \mathrm{C}$. anéantit tous les germes, et à $100-110^{\circ} \mathrm{C}$. , le lait ne contient que quelques spores des bactéries d'espèce $B$. Subtilis et B. mesentericus.

$2^{\circ}$ Le lait pasteurisé à $85^{\circ} \mathrm{C}$. et déposé dans des bouteilles en lieu chaud se gâte par la fermentation, ou butyrique, ou putrifiactive, et étant consommé, il peut produire des accidents gastriques ou intestinaux.

$3^{\circ}$ La lait stérile se conserve un temps illimité dans des flacons bien fermés, et dans le cas où il contiendrait quelques spores d'espèce de B. mesentericus, sa conservation est pourtant plus longue que celle du lait 
pasteurisé, surtout si on le maintient à une température au-dessous de $12^{\circ} \mathrm{C}$., parce que les bactéries ci-dessus mettent dans ces conditions un temps fort long à se développer.

$4^{\circ} \mathrm{A}$ ces avantages, on peut ajouter en ce qui concerne la machine de Nielsen, le fait que le chauffage et le refroidissement se font à l'abri de l'air. Le lait contient des vitamines qui sont endommagées, non seulement par le chauffage, mais surtout pendant le refroidissement, par le contact de l'oxygène de l'air avec le lait. FrederICIA (1) a démontré cet avantage par des essais d'après lesquels le lait uielsenisé a conservé les vitamines dans les proportions des $3 / 4$.

Ces observations montrent en définitive que le stérilisateur de Nielsen donne par le chauffage à $128-130^{\circ} \mathrm{C}$. un lait parfaitement stérile, à condition de stériliser convenablement les cylindres réfrigérants pendant le nettoyage.

\section{NOUVELLES RECHERCHES SUR LA PASTEURISATION A BASSE TEMPÉRATURE}

A) Facteurs qui influent sur la destruction par la chaleur du bacille tuberculeux dans le lait (1);

B) Résistance à la chaleur du bacille tuberculeux dans le lait (2). par le Dr K. KATRANDJIEFF

Chef de service à l'Institut vétérinaire bactériologique de Sofia.

Les premières recherches de Yersin (3) en 1888, puis de Grancher et Ledoux-Lebard (4) en 1892 sur l'influence de la chaleur sur le bacille tuberculeux cultivé dans des milieux de bouillon glycériné sont considérées comme classiques dans l'étude de l'action de la chaleur sur la destruction ou l'atténuation du bacille de Koch. Ces auteurs ont constaté que la virulense des bacilles diminue lorsqu'on les porte à $60^{\circ} \mathrm{C}$. pendant 5 minutes; une température de $70^{\circ} \mathrm{C}$. pendant 5 minutes détruit toute virulence et toute vitalité.

Ces considérations ont ouvert la voie à de nombreuses applications pratiques. De nombreux travaux ont suivi dont quelques-uns doivent retenir l'attention.

Forster (5) vient de démontrer que le lait tuberculeux, ou le lait infecté artificiellement, chauffé à $55^{\circ} \mathrm{C}$. pendant 4 heures, à $60^{\circ} \mathrm{C}$. pendant une heure, à $65^{\circ} \mathrm{C}$. pendant 15 minutes, etc., perd sa virulence. D'un autre côté, Morgenroth (6) prétend avoir constaté que le chauffage d'un lait tuberculeux à $70^{\circ} \mathrm{C}$. pendant 30 minutes détruit toute virulence. Levr et BRuns (7) se sont servis de lait additionné de bacilles tuberculeux très virulents; ils le portent à $65^{\circ} \mathrm{C}$. pendant 15 ou 25

(1) Fredericta. - Physiologieal papers, Aarhus, 1926. 